\title{
Assessment of Active Case Search of COVID-19 in Healthcare Facilities during the Third Wave of the Pandemic in Ekiti State, Nigeria
}

\author{
Eyitayo Ebenezer Emmanuel, Ayomide Oluwaseyi Aibinuomo, Tope Michael Ipinnimo*, \\ Mary Oluwasolape Ilesanmi, John Ayodeji Adu
}

WHO Field Office, Ado-Ekiti, Ekiti State

Email: eyitayoe@yahoo.com, ayoaibinuomo@gmail.com, ^abbeymagnus@yahoo.com, sholapejo@gmail.com, ayodejijohn7@gmail.com

\begin{abstract}
How to cite this paper: Emmanuel, E.E. Aibinuomo, A.O., Ipinnimo, T.M., Ilesanmi, M.O. and Adu, J.A. (2022) Assessment of Active Case Search of COVID-19 in Healthcare Facilities during the Third Wave of the Pandemic in Ekiti State, Nigeria. Journal of Biosciences and Medicines, 10 , 10-19.
\end{abstract}

https://doi.org/10.4236/jbm.2022.102002

Received: January 2, 2022

Accepted: January 27, 2022

Published: January 30, 2022

Copyright $\odot 2022$ by author(s) and Scientific Research Publishing Inc. This work is licensed under the Creative Commons Attribution International License (CC BY 4.0).

http://creativecommons.org/licenses/by/4.0/

\section{(c) (i) Open Access}

\begin{abstract}
Early case detection and isolation of patients infected with highly infectious diseases through active case search (ACS) are important for early commencement of treatment and control of the disease within a population. This study aimed to assess the ACS of COVID-19 in healthcare facilities during the third wave of the pandemic in Ekiti State, Nigeria. This cross-sectional study was conducted in all healthcare facilities in Ekiti State. All cadres of local government primary healthcare workers were trained to carry out the ACS using semi-structured interviewer-administered questionnaire uploaded on android phone software open data kit (ODK). ACS was conducted between June and September (epidemiological week 23 to 36), 2021 and the collected data were analyzed using IBM SPSS version 23.0. P-value $<0.05$ was taken as significant. The number of ACS visits for COVID-19 increases with epidemiological week progression, but the highest was in week 32 (9.9\%). Cases of suspected COVID-19 in the healthcare facilities in the last one week were reported in more than half $(58 \%)$ of the visits and $57.8 \%$ of those reported, documented between 1 - 4 cases. Also, out of those that were reported, $42 \%$ of them missed (not line listed) at least one suspected case of COVID-19. The private health facilities missed more cases of COVID-19 (54.3\%) than other categories of health facilities $(\mathrm{p}<0.001)$. The health educators and local government immunization officers (LIO) than other cadre of healthcare workers found most missed cases $(57.1 \%$ and $51.4 \%$ respectively) in their visits $(\mathrm{p}<0.001)$. The health educator as well as the LIO detected the highest proportion of missed suspected cases which shows that disease surveillance officers alone may not be adequate for ACS, there is a need to involve other local government team member.
\end{abstract}




\section{Keywords}

Active Case Search, COVID-19, Health Care Facilities, Ekiti State

\section{Introduction}

Corona virus disease (COVID-19) is an infectious disease caused by the severe acute respiratory syndrome coronavirus 2 (SARS-CoV-2) [1]. The first confirmed case of the disease was discovered in Nigeria on 27th February 2020 by Nigeria Center for Disease Control (NCDC), [2] following this, 208,630 cases have been confirmed with 2767 deaths as of 15th October 2021. [3] The advent of the delta variant of SARS-CoV-2 poses a great deal of problem in Nigeria and other affected countries. The delta variant is recognized by the World Health Organization (WHO) as a variant of concern, given its increased transmissibility. The variant has been detected in over 90 countries and is expected to spread to more countries. The variant has also been linked to a surge in cases in nations where it is the dominant strain in circulation and it has been implicated in the third wave of COVID-19 disease [4].

Active case search (ACS) is a systematic search for symptomatic cases of an infectious disease using a specified case definition and has been used in infectious diseases such as poliomyelitis; its use in COVID-19 pandemic third wave has not been well documented [1]. ACS is an important approach for capturing all the cases, as many may not have access to healthcare facilities and may seek care elsewhere during outbreaks of disease especially in pandemic season of COVID-19 [5] [6]. Early case detection and isolation of patients infected with highly infectious diseases are important for early commencement of supportive treatment and control of further transmission of the disease within a population [1] [7] [8].

ACS is a key surveillance activity for control of epidemic prone diseases such as COVID-19, which relies on active early identification of suspected cases which may not otherwise be reported [9] [10]. ACS teams may visit households or health facilities to look for possible COVID-19 cases [11]. Health facilities provide an appealing location for ACS based on symptoms screening. Due to the severity of symptoms individuals with COVID-19 are likely to come in contact with the healthcare system during the course of their illness. These cases may however not be reported due to lack of awareness of signs and symptoms of COVID-19 and the procedure for reporting suspected cases [12].

The COVID-19 virus is rapidly evolving [13] and such evolution requires continuous watch and scrutiny at all levels. A robust comprehensive surveillance, once in place, should be maintained even in areas where transmission has been suppressed or controlled, even if there are few or no cases. However, given the time required for adequate immunization coverage in the population at large, subsequent pandemic waves are anticipated and detection methods for SARS-CoV-2 
remain a crucial part of containment and mitigation strategies [14] [15].

There is paucity of study on ACS of COVID-19 with only few literature reports on it. This study is the first of its kind in this environment and would serve as a source of baseline information on ACS of the disease. This study therefore aimed to assess the ACS (through number of visits) of COVID-19 in healthcare facilities by epidemiological weeks, local government healthcare workers and health facility type during the third wave of the pandemic in Ekiti State, Nigeria.

\section{Methodology}

This study is a cross-sectional study carried out between June (Epidemiological week 23) and September (Epidemiological week 36), 2021 in all the healthcare facilities in Ekiti State, Nigeria. Ekiti State is located in the south-western part of the country with three senatorial districts and sixteen Local Government Areas (LGAs). The state has an estimated total population size of 3,816,784 (2021 projected population) [16].

The study was carried out by trained local government healthcare workers such as the medical officer of health $(\mathrm{MOH})$ and primary healthcare coordinators (PHCC), health educators, public health nurses, local immunization officers (LIO), disease surveillance officers (disease surveillance and notification officers-DSNO and assistant disease surveillance and notification officers-ADSNO) among others who visited all the healthcare facilities (primary, secondary, tertiary and private) in their LGA of jurisdiction once per week on ACS of COVID-19.

The COVID-19 suspected case definitions used in this research is the updated case definition by the NCDC released in January 2021 with the following four options:

Option A: Any person who meets the clinical and epidemiological criteria:

Clinical criteria: Acute onset of any two or more of the following signs or symptoms: fever, cough, runny nose, sore throat/pharyngitis, headache, difficulty breathing/dyspnea, nausea, loss of taste, loss of smell, general weakness/fatigue, diarrhea, chest pain, vomiting, chills/sweating, muscle pain/myalgia, wheezing, abdominal pain, altered mental status.

Epidemiological criteria: 1. Residing or working in a setting with high risk of transmission of the virus: for example, closed residential settings and humanitarian settings, such as camp and camp-like settings for displaced persons, any time within the 14 days before symptom onset;

OR 2. Residing in or travel to a country, state, LGA with community transmission as classified by the NCDC, anytime within the 14 days before symptom onset;

OR 3. Working in health setting, including within health facilities anytime within the 14 days before symptom onset.

Option B: A patient with severe acute respiratory illness (SARI: acute respiratory infection with history of fever or measured fever of $\geq 38^{\circ} \mathrm{C}$; and cough; with 
onset within the last 10 days; and who requires hospitalization) or with chest imaging showing findings suggestive of COVID-19 disease.

Option $C$ : A person not meeting epidemiological criteria with a positive SARS-CoV-2 antigen-detecting rapid diagnostic test (Ag-RDT), irrespective of any symptoms.

Option D: Close contact of a confirmed case, irrespective of any symptoms.

Anyone of the four options A to D qualifies as a suspected COVID-19 case. Using this case definition of suspected COVID-19 case, the trained local government healthcare workers reviewed all medical records within the last one week in the health facilities. Symptoms and signs of COVID-19 from the suspected case definition were then assessed whether to validate these as suspected COVID-19 case based on the clinical features and epidemiological link of the patient. The team also assessed healthcare facilities workers knowledge of suspected case definition of COVID-19 and the process for notifying. Upon visit to the health facilities, the trained local government healthcare workers presented themselves to the officer in charge (OIC) of the health facilities to discuss their visit and to request designated focal points where medical records would be reviewed and ACS could be carried out. These trained local government healthcare workers started by asking questions on signs and symptoms of COVID-19, if anyone has presented within the last week with such feature, they also go through the out-patient register of the health facilities to see if there is any case that meets the suspected case definition of COVID-19 in the last one week. Cases that meet the suspected case definition were then contacted and confirmatory tests carried out. Confirmed cases were then treated as per protocol based on severity of symptoms.

Semi-structured interviewer-administered questionnaire uploaded on android phone software open data kit (ODK) was used to collect information at the health facilities. Data collected by the trained local government healthcare workers included: the epidemiology week of the visit, health facility type, knowledge of the suspected case definition by the healthcare workers in the health facilities, cases of suspected COVID-19 seen in the healthcare facilities in last one week and cases of suspected COVID-19 missed (not line listed) in the healthcare facilities in last one week. The WHO epidemiological week calendar was used to determine the week based on the date of the visit. Health facilities were categorized based on type into public primary, public secondary, public tertiary and private health facilities. Collected data were downloaded from the ODK and analyzed using IBM SPSS version 23.0. Frequency and percentages were presented in tables at univariate level of analysis. Chi-square was used to assess the association between ACS and independent variables. P-value $<0.05$ was taken as significant.

Ethical approval was sought and obtained from the research and ethics review committee of Federal Teaching Hospital, Ido-Ekiti, Ekiti State, Nigeria. Participation was anonymous and voluntary. Informed consent was taken from the OIC of the health facilities. 


\section{Results}

\subsection{Active Case Search Conducted by Epidemiological Week, Designation and Health Facility Type}

A total of 2640 ACS were conducted within the fourteen (14) epidemiological week period (i.e. epidemiological week 23 to 36). The highest number of ACS were carried out in week 32 with 261 (9.9\%) ACS. Weeks 31, 33, 27 and 29 also recorded high ACS with 250 (9.5\%), 243 (9.2\%), 238 (9.0\%) and 232 (8.8\%) ACS respectively. However, the least number of case search was in week 23 with 32 ACS representing 1.2\% of the entire period case searches (Figure 1).

About one-quarter of the entire case search were done by the disease surveillance officers (DSNOs and ADSNO), this is followed by the MOH/PHCC with 340 (12.9\%) ACS and laboratory scientist with 328 (12.4\%) ACS. Environmental health officers conducted the least ACS activity with 244 (9.2\%) ACS.

Majority $(87.2 \%)$ of the ACS were carried out at the primary health facilities, followed by secondary health facilities with $7.7 \%$ of cases. The private health facilities accounted for $4.5 \%$ of the cases while the tertiary health facilities contributed only $0.6 \%$ of the COVID-19 ACS (Table 1 ).

\subsection{Active Case Search}

Cases of suspected COVID-19 in the healthcare facilities in last one week were reported in more than half (58\%) of the visits and $57.8 \%$ of those reported, documented between 1 - 4 cases. Also, out of those that were reported, $42 \%$ of them missed (not line listed) at least one suspected case of COVID-19. Majority (98.5\%) of the health care workers know about the standard case definition of COVID-19 as against only $1.5 \%$ of them who don't know the disease case definition (Table 2).

\subsection{Relationship between Healthcare Facilities Type and Active Case Search}

All (100\%) the healthcare workers in the tertiary health facilities know about the standard case definition of COVID-19, this is followed by the healthcare workers in the secondary health facilities (99.0\%) primary health facilities (98.5\%) and private health facilities (96.7\%). However, this differences in the knowledge of COVID-19 case definition was not statistically significant $(\mathrm{p}=0.349)$.

The private health facilities missed more cases of COVID-19 (54.3\%) than other categories of health facilities, with the secondary health facilities having the least missed cases $(23.6 \%)$. This difference is statistically significant ( $p<$ 0.001) (Table 3).

\subsection{Relationship between Designation of Healthcare Workers and Active Case Search}

The laboratory scientist had the least number of missed cases (29.9\%) out of the cases searched by them while the health educators and LIO found most missed 
cases $(57.1 \%$ and $51.4 \%$ respectively) in their visits. The difference of missed cases along the designation of healthcare workers was statistically significant ( $\mathrm{p}$ $<0.001$ ) (Table 4).

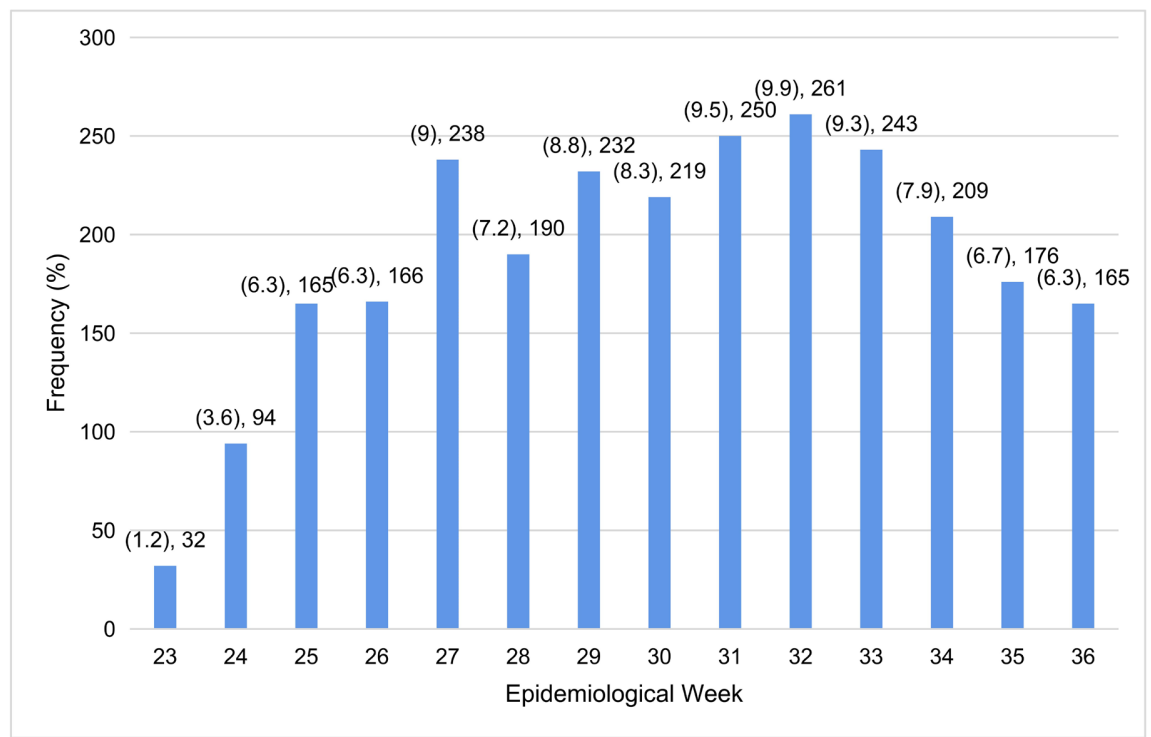

Figure 1. Active case search conducted by epidemiological week.

Table 1. Active case search conducted by designation and health facility type.

\begin{tabular}{|c|c|c|}
\hline Variable & Frequency $(N=2640)$ & Percent (\%) \\
\hline \multicolumn{3}{|l|}{ Designations } \\
\hline Disease Surveillance Officers & 590 & 22.3 \\
\hline Environment Health Officers & 244 & 9.2 \\
\hline Health Educator & 281 & 10.6 \\
\hline Laboratory Scientist & 328 & 12.4 \\
\hline LIO & 251 & 9.5 \\
\hline $\mathrm{MOH} / \mathrm{PHCC}$ & 340 & 12.9 \\
\hline Public Health Nurses & 313 & 11.9 \\
\hline State Supervisors & 293 & 11.1 \\
\hline \multicolumn{3}{|l|}{ Health facility type } \\
\hline Primary & 2302 & 87.2 \\
\hline Secondary & 203 & 7.7 \\
\hline Tertiary & 15 & 0.6 \\
\hline Private & 120 & 4.5 \\
\hline
\end{tabular}

LIO-local government immunization officer, MOH/PHCC-medical officer of health/primary healthcare coordinator. 
Table 2. Active case search.

Variable

Frequency $(\mathrm{N}=2640)$

Percent (\%)

Knowledge of the suspected case definition by the healthcare workers in the health facilities

$\begin{array}{lcr}\text { Yes } & 2600 & 98.5 \\ \text { No } & 40 & 1.5\end{array}$

Cases of suspected COVID-19 in the healthcare facilities in last one week

$\begin{array}{lll}\text { Yes } & 1531 & 58.0 \\ \text { No } & 1109 & 42.0\end{array}$

Cases of suspected COVID-19 seen in the healthcare facilities in last one week $(n=1531)$

$1-4$

886

Greater than 4

645

42.1

Cases of suspected COVID-19 missed (not line listed) in the healthcare facilities in last one week $(n=1531)$

$\begin{array}{lll}\text { No missed case } & 892 & 58.3\end{array}$

At least one case missed

639

Table 3. Relationship between healthcare facilities type and active case search.

Active Case Search

Health facility type

Primary Secondary Tertiary Private $\chi^{2} \quad$ p-value

Knowledge of the standard suspected case definition by the healthcare workers in the health facilities $(\mathrm{N}=2640)$

\begin{tabular}{|c|c|c|c|c|c|c|}
\hline Yes & 2268 (98.5) & $201(99.0)$ & $15(100.0)$ & $116(96.7)$ & $3.294^{\mathrm{a}}$ & 0.349 \\
\hline No & $34(1.5)$ & $2(1.0)$ & $0(0.0)$ & $4(3.3)$ & & \\
\hline
\end{tabular}

Cases of suspected COVID-19 missed (not line listed) in the healthcare facilities in last one week $(n=1531)$

$\begin{array}{lcccccc}\text { No missed case } & 717(56.3) & 133(76.4) & 10(71.4) & 32(45.7) & 31.133 & <0.001 \\ \text { least one case missed } & 556(43.7) & 41(23.6) & 4(28.6) & 38(54.3) & \end{array}$

$\chi^{2}$-chi square.

Table 4. Relationship between designation of healthcare workers and active case search.

\begin{tabular}{|c|c|c|c|c|}
\hline \multirow[t]{2}{*}{ Designations } & \multicolumn{4}{|c|}{$\begin{array}{l}\text { Cases of suspected COVID-19 missed (not line listed) } \\
\text { in the healthcare facilities in last one week }(n=1531)\end{array}$} \\
\hline & No missed case & At least one missed case & $x^{2}$ & p-value \\
\hline Disease Surveillance Officers & $194(58.6)$ & $137(41.4)$ & $37.440^{\mathrm{a}}$ & $<0.001$ \\
\hline Environment Health Officers & $62(56.4)$ & $48(43.6)$ & & \\
\hline Health Educator & $67(42.9)$ & $89(57.1)$ & & \\
\hline Laboratory Scientist & $148(70.1)$ & $63(29.9)$ & & \\
\hline LIO & $70(48.6)$ & $74(51.4)$ & & \\
\hline $\mathrm{MOH} / \mathrm{PHCC}$ & $123(57.2)$ & $92(42.8)$ & & \\
\hline Public Health Nurses & $102(59.3)$ & $70(40.7)$ & & \\
\hline State Supervisors & $126(65.6)$ & $66(34.4)$ & & \\
\hline
\end{tabular}

LIO-local government immunization officer, MOH/PHCC-medical officer of health/primary healthcare coordinator, $\chi^{2}$-chi square. 


\section{Discussion}

The overall case search during 14-week period of the epidemiological week was 2640 cases. This number increases with progression in the week except for a minimal reduction towards the last 3 weeks. The steady increase earlier seen might be due to the increase in awareness on ACS of COVID-19 as the week progresses while the late reduction seen may be due to reduced COVID-19 cases as well as fatigue by the healthcare workers. This progressive increase is comparable with the weekly increase in incidence of COVID-19 in Africa continent during the fourth wave of the disease [17].

The disease surveillance officers (DSNOs and ADSNO) conducted more of the ACS activities accounting for about one-quarter of the total case search as against the remaining three quarters done by seven other different cadres of healthcare workers. Active case search is a major part of terms of reference of the disease surveillance officers and as such may account for this finding. The finding may also be due to the level of knowledge obtained from several trainings and expertise developed by disease surveillance officers in disease search activities over time [18].

A substantive majority of the ACS (87.2\%) for COVID-19 were done at the primary healthcare facilities in the state. This is unexpected looking at the high numbers of primary healthcare facilities in the state and also putting in mind that this level of healthcare is the first point of contact for most members of the community [19].

Almost all the healthcare workers (98.5\%) know about the standard case definition of COVID-19. This finding is good for a more effective case search and better outcome. However, most of the few who were unfamiliar with the definition were in the private hospital showing the need for more technical assistance to these facilities. Also, the private health facilities also missed more suspected cases of COVID-19 (more than half of the cases seen at these health facilities) compared to other healthcare facilities. This may be due to lesser numbers of qualified staff at the private healthcare facilities and also lesser exposure to training and expertise in detecting suspected cases of the disease. This high missed case rate may water down the efforts towards detection of COVID-19 and more technical assistance to the private healthcare facilities will help to further strengthen effective case search [18].

Furthermore, among the cadre of healthcare workers, the health educators and LIOs detected the highest proportion of missed suspected cases. This outcome is better than even the proportion among the disease surveillance officers and the medical officers of health. This may further strengthen the need to involve local government health educator and LIOs in ACS for COVID-19 and other priority disease in future. It also emphasized the need for team work at the primary healthcare level such that ACS would not be left for the disease surveillance officers alone.

This study is limited by the fact that it was health facility based. There are 
many cases of COVID-19 that may not be present at the health facility and as such would not be captured by the study. Future research may focus on comprehensive ACS which will involve search in both the health facility and the community.

In conclusion, the number of ACS increases with progression in the epidemiological week but efforts must be put in place for sustenance of this progress to avoid waning off seen towards the tail end of the weeks. Also, the private health facilities with lower knowledge of the standard case definition had a higher proportion of missed cases of the disease, this may suggest that better knowledge and understanding of the standard case definition may help detection of more suspected cases and reduce missed cases. Lastly, the health educator and LIO detected the highest proportion of missed suspected cases which shows that disease surveillance officers alone may not be adequate to conduct ACS, other LGA teams should be carried along and actively involved.

\section{Conflicts of Interest}

The authors declare no conflicts of interest regarding the publication of this paper.

\section{References}

[1] Fasina, F.O., Shittu, A., Lazarus, D., Tomori, O., Simonsen, L., Viboud, C., et al. (2014) Transmission Dynamics and Control of Ebola Virus Disease Outbreak in Nigeria. Eurosurveillance, 19, 20920. https://doi.org/10.2807/1560-7917.ES2014.19.40.20920

[2] Nigeria Centre for Disease Control (2021) An Update of COVID-19 Outbreaks in Nigeria for Week 9_29022020. https://ncdc.gov.ng/reports/weekly

[3] Nigeria Centre for Disease Control (2021) Situation Report on COVID 19 in Nigeria. https://covid19.ncdc.gov.ng/\#

[4] Nigeria Centre for Disease Control (2021) Confirmed Case of Delta Variant Detected in Nigeria.

https://ncdc.gov.ng/news/333/confirmed-case-of-delta-variant-detected-in-nigeria

[5] World Health Organization (2015) Ebola in West Africa: Learning the Lessons. The Lancet, 385, 591-592. https://doi.org/10.1016/S0140-6736(15)60075-7

[6] Yamin, D., Gertler, S., Ndeffo-Mbah, M.L., Skrip, L.A., Fallah, M., Nyenswah, T.G., et al. (2015) Effect of Ebola Progression on Transmission and Control in Liberia. Annals of Internal Medicine, 162, 11-17. https://doi.org/10.7326/M14-2255

[7] Faye, O., Boëlle, P.Y., Heleze, E., Faye, O., Loucoubar, C., Magassouba, N.F., et al. (2015) Chains of Transmission and Control of Ebola Virus Disease in Conakry, Guinea, in 2014: an Observational Study. The Lancet Infectious Diseases, 15, 320-326. https://doi.org/10.1016/S1473-3099(14)71075-8

[8] Drake, J.M., Kaul, R.B., Alexander, L.W., O’Regan, S.M., Kramer, A.M., Pulliam, J.T., et al. (2015) Ebola Cases and Health System Demand in Liberia. PLoS Biology, 13, 12-19. https://doi.org/10.1371/journal.pbio.1002056

[9] World Health Organization (2020) Public Health Surveillance for COVID-19: Interim Guidance, 7 August 2020. https://apps.who.int/iris/handle/10665/333752

[10] BMJ Best Practices (2018) Ebola Virus Infection: Screening 2018. 
https://bestpractice.bmj.com/topics/en-us/1210/screening

[11] Blackley, D.J., Lindblade, K.A., Kateh, F., Broyles, L.N., Westercamp, M., Neatherlin, J.C., et al. (2015) Rapid Intervention to Reduce Ebola Transmission in a Remote Village-Gbarpolu County, Liberia, 2014. MMWR Morb Mortal Wkly Rep. ; 64: 175-8.

[12] Matanock, A., Arwady, M.A., Ayscue, P., Forrester, J.D., Gaddis, B., Hunter, J.C., et al. (2014) Ebola Virus Diseases Cases among Health Care Workers Not Working in Ebola Treatment Units-Liberia, June-August, 2014. Morbidity and Mortality Weekly Report, 63, 1077-1081.

[13] Wang, L., Bi, Y.H. and Gao, G.F. (2020) Epidemiological Model Suggests D614G Spike Protein Mutation Accelerates Transmission of COVID-19-Worldwide, 2020. China CDC Weekly, 2, 946-947. https://doi.org/10.46234/ccdcw2020.247

[14] Sharma, O., Sultan, A.A., Ding, H. and Triggle, C.R. (2020) A Review of the Progress and Challenges of Developing a Vaccine for COVID-19. Frontiers in Immunology, 14, 585354. https://doi.org/10.3389/fimmu.2020.585354

[15] Ortiz-Prado, E., Simbaña-Rivera, K., Gómez-Barreno, L., Rubio-Neira, M., Guaman, L.P., Kyriakidis, N.C., et al. (2020) Clinical, Molecular, and Epidemiological Characterization of the SARS-CoV-2 Virus and the Coronavirus Disease 2019 (COVID-19), a Comprehensive Literature Review. Diagnostic Microbiology and Infectious Disease, 98, 115094. https://doi.org/10.1016/j.diagmicrobio.2020.115094

[16] Ekiti State Government (2016) History of Ekiti State. http://ekitistate.gov.ng

[17] World Health Organization (2021) Weekly Epidemiological Update on COVID19-14 December 2021; Edition 70: 1-2.

https://www.who.int/publications/m/item/weekly-epidemiological-update-on-covid -19---14-december-2021

[18] Commission on Social Determinants of Health (2008) Closing the Gap in a Generation: Health Equity through Action on the Social Determinants of Health (Knowledge, Monitoring, and Skills: The Backbone of Action) Chapter 16; p.176-191. https://www.who.int/social_determinants/final_report/csdh_finalreport_2008.pdf

[19] World Health Organization (2021) Fact Sheet/PHC. https://www.who.int/news-room/fact-sheets/detail/primary-health-care 